\title{
A Computational Procedure for Generating Specimens of BIM and Point Cloud Data for Building Change Detection
}

\author{
Ling Ma ${ }^{1}$, Rafael Sacks ${ }^{2}$, Reem Zeibak-Shini ${ }^{3}$ and Sagi Filin ${ }^{4}$ \\ ${ }^{1}$ Postdoctoral Researcher, National Building Research Institute, Technion, Haifa \\ 32000, Israel+972-4-8292245, lingma@,technion.ac.il \\ ${ }^{2}$ Assoc. Prof., Head Dept. of Structural Engineering and Construction Management, \\ Technion, Haifa 32000, Israel +972-4-8293190, cvsacks@technion.ac.i1 \\ ${ }^{3} \mathrm{PhD}$ Candidate, National Building Research Institute, Technion, Haifa 32000, Israel \\ +972-4-8293120, reemz@technion.ac.il \\ ${ }^{4}$ Assoc. Prof., Head Dept. of Transport Engineering and Geo-Informatics, Technion, \\ Haifa 32000, Israel +972-4-8295855, filin@technion.ac.il
}

\begin{abstract}
The potential for automated construction quality inspection, construction progress tracking and post-earthquake damage assessment drives research in interpretation of remote sensing data and compilation of semantic models of buildings in different states. However, research efforts are often hampered by a lack of full-scale datasets. This is particularly the case for earthquake damage assessment research, where acquisition of scans is restricted by scarcity of access to post-earthquake sites. To solve this problem, we have developed a procedure for compiling digital specimens in both pre- and post-event states and for generating synthetic data equivalent to which would result from laser scanning in the field. The procedure is validated by comparing the physical and synthetic scans of a damaged beam. Interpretation of the beam damage from the synthetic data demonstrates the feasibility of using this procedure to replace physical specimens with digital models for experimentation and for other civil engineering applications.
\end{abstract}

Keywords: Computational procedure; Laser scanning; BIM; Damage assessment; Change detection.

\section{INTRODUCTION}

If 'as-designed', 'as-built' and 'as-is' BIM (Building Information Modeling) models can be compiled to represent the design, construction and maintenance phases of a building project, then comparison of the models can serve different use-cases, such as:

- construction progress tracking and quality checking can be abstracted as change detection between 'as-designed' and 'as-built' states of the building, and 
- disaster damage assessment and structural health monitoring can be abstracted as change detection between 'as-built' and 'as-is' states of the building.

Remote sensing technologies, including terrestrial laser scanning (TLS), photo/videogrammetry, etc., are potentially efficient and effective surveying tools that can facilitate detection of changes among the different states of a building. Bosché et al. (2014) explored the opportunity for frequent, detailed and semantically rich assessment of as-built status of construction projects by joining point cloud data (PCD) and 3D/4D BIM models that describe mechanical, electrical and plumbing (MEP) works. Akinci et al. (2006) proposed a formalism for using advanced sensor systems and integrated project models for active construction quality control. Kashani et al. (2014) used PCD acquired by TLS before and after a tornado to evaluate how much damage was caused to buildings. German et al. (2013) developed an approach based on real-time analysis of video frames to identify the cracks in concrete columns and other structural elements. Torok et al. (2014) used images obtained from an unmanned robotic platform to similarly identify cracks and main structural elements. All of these share the common thread of comparison of buildings at different stages of their life-cycle.

Research and development (R\&D) of such change detection systems commonly defines their function as consisting of three major steps:

1) Choosing building specimens, scanning them and preparing point cloud data.

2) Semantic interpretation of the resulting PCD, with or without information from a BIM model of an earlier state.

3) Identification and interpretation of the changes between the current and previous states of the building. This stage may be performed with PCD or with BIM information, and sometimes both.

However, R\&D of the change detection systems has been often restricted by the lack of available remote sensing data and building specimens. This is particularly so for the case of earthquake damage assessment research, where acquisition of remote sensing data and models is restricted by the scarcity of access to the post-earthquake sites. Specimens of earthquake damaged buildings or components can be reproduced by large-scale shaking tables (Kasai et al. 2010; van de Lindt et al. 2010; Panagiotou, Restrepo, and Conte 2010), e.g., LHPOST, the large high performance outdoor shake table at the Univ. of California, San Diego (Conte et al. 2004), and E-Defense, the world's largest shaking table to date at the Hyogo Earthquake Engineering Research Center (Tagawa and Kajiwara 2007). However, such large scale experiments have prohibitive cost and produce single specimens with each test. Thus for the purposes of R\&D of change detection systems, a practical approach is needed for preparing multiple highly detailed building specimens with low cost. 
In addition, there are no open repositories of PCD of damaged buildings with sufficient resolution (i.e. resolution of TLS). Sample data of earthquake sites are available at the lower resolution of airborne and space-based imagery for site-scale monitoring.

To overcome these problems, we have developed a computational procedure for preparing datasets that include BIM models of different states of a building, synthetic TLS PCD sets that can be used for development and rigorous testing of change detection systems. This paper presents a validation of the computational procedure and demonstrates the feasibility of its use as a substitute for physical experiments. The following section describes the procedure. In section 3, the procedure is validated by comparing the field scan of a damaged reinforced concrete beam with the synthetic scan of the BIM model of the same damaged beam. In section 4, feasibility is further demonstrated by comparing the semantic interpretation results obtained from interpreting the synthetic PCD set with the results from the real PCD set of the same damaged beam.

\section{PROCEDURE FOR PREPARING SYNTHETIC SAMPLE DATA}

\section{BIM model as digital specimen}

The computational procedure begins with compiling BIM models of a building in its different states. BIM is a process of producing 3D building models that represent a building and its components together with the components' functional characteristics. It is broadly used to provide semantically rich representation of design intent (Eastman et al. 2011). It is applicable over the whole life-cycle of a building. A recent extension proposed for the IFC standard open BIM data schema (BuildingSmart 2013) lays the groundwork for modeling of the damaged state of building components (Ma, Sacks, and Zeibak-Shini 2014). The user begins by modeling the 'as-built' state of a building from engineering drawings. Next, the user models the 'as-damaged' state of the building by examining photos of the building taken after the earthquake event. The 'as-damaged' state is compiled in the BIM software by performing CSG clipping and displacement operations on the 'as-built' BIM model of the building, thus ensuring that the component dimensions are correct. However, the objects that result from CSG clipping are represented as separate building components, rather than as the parts of one damaged component. As a result, the model must be semantically enriched using custom-built software that parses the IFC file of the model and encodes the identity of each original component to the corresponding objects that represent its damaged state.

The damaged reinforced concrete beam shown in Figure 1 was used for testing and validation of the procedure. The beam was damaged in unrelated research at the National Building Research Institute (NBRI), Technion, Haifa (Schwarz, Hanaor, 
and Yankelevsky 2008). Figure 1 also shows an 'as-damaged' BIM model that was prepared to model the beam.

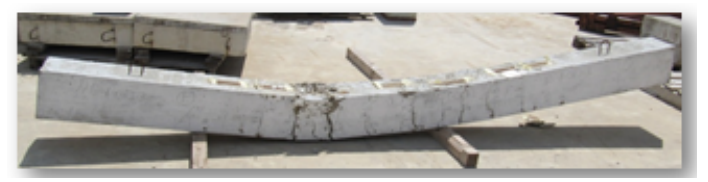

(a)

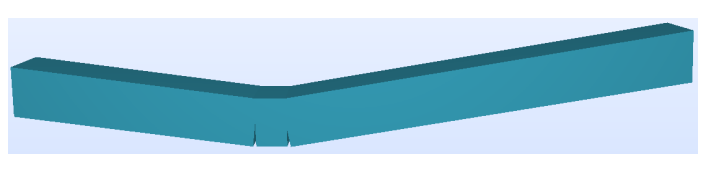

(b)

Figure 1. (a) Photo of a damaged beam prepared at NBRI, (b) 'as-damaged' BIM model of the beam.

\section{Laser scanning emulator}

TLS is a 3D surveying technology that has been in practical application since the mid-to-late 1990s. The attractiveness of such a system can be explained by its ability to provide directly dense and accurate 3D point-clouds without physical access to the studied area. Its ability to rapidly capture highly accurate wide three-dimensional regions without being influenced by scene complexity, makes it appropriate for reliable detection of spatial changes.

In order to generate PCD samples, we implemented a laser scanning emulator (Ma et al. 2014). From a BIM model, the emulator can generate synthetic PCD in a manner similar to the way that TLS works in the field. The emulator transmits virtual laser beams from a fixed position/viewpoint to 'hit' the 3D model in a given direction. The scanning direction is defined by two parameters, i.e., pan and tilt angle, in a manner similar to that in which the laser beam deflection unit of a physical scanner moves. The emulator traverses all the pan and tilt angles to collect the closet intersection of the virtual laser beam to the 3D model, resulting in synthetic PCD. The emulator software can also introduce range inaccuracy and position inaccuracy to the synthetic data to simulate the measurement noise present in field scans.

The damaged beam (Figure 1a) was scanned using a Leica ScanStation C10 (LEICA 2014) at NBRI. A synthetic scan of the 'as-damaged' BIM model was performed from the same viewpoint as the field scan using the custom-built emulator (Figure 1b). The PCD sets from the field and the synthetic scans are compared in Figure 2.

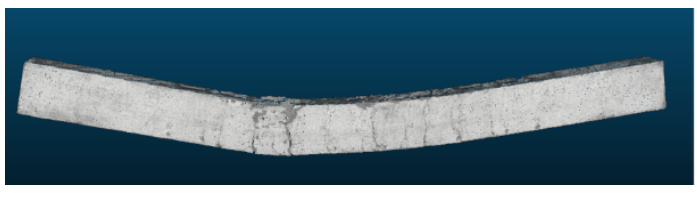

(a)

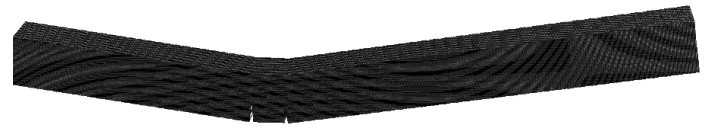

(b)

Figure 2. (a) Field scan, (b) synthetic scan of the damaged beam. 


\section{VALIDATION OF THE SYNTHETIC SAMPLE}

The first validation of the 'as-damaged' BIM model and the synthetic PCD resulting from the emulator is performed by comparing the point clouds directly. This tests both the modeling accuracy and the scanning emulation in a single step, thus testing the computational procedure as a whole. A minor drawback is that it is not possible to automatically distinguish the source of any error.

The PCD is usually represented using Euclidian coordinates of 3D points (i.e., X, Y, $Z$ ) in which the points are irregularly spaced. In a spherical coordinate system, where the points are represented as pan angle, tilt angle and range distance, the data is more conveniently organized because the angular spacing of the scanning process is fixed. The data can be interpreted as $2.5 \mathrm{D}$ range image whose image intensity values represent the range distances. Thus due to the regularity of the representation, when the range images of two PCDs are aligned, the differences between the two datasets can be derived directly from the differences of the intensity values or range distances (Zeibak and Filin 2007).

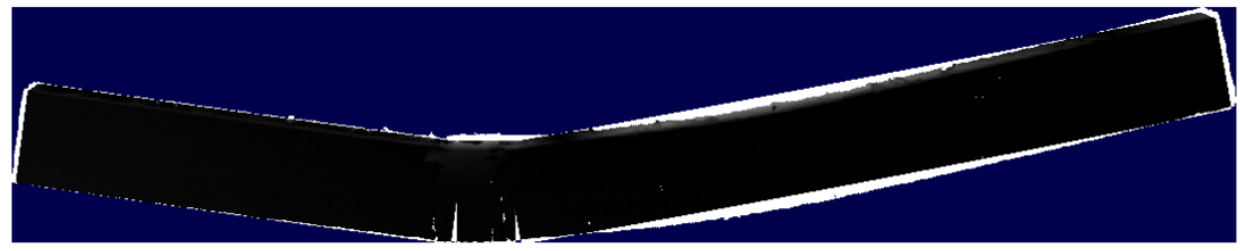

Figure 3. Range difference image of the synthetic and the physical scan

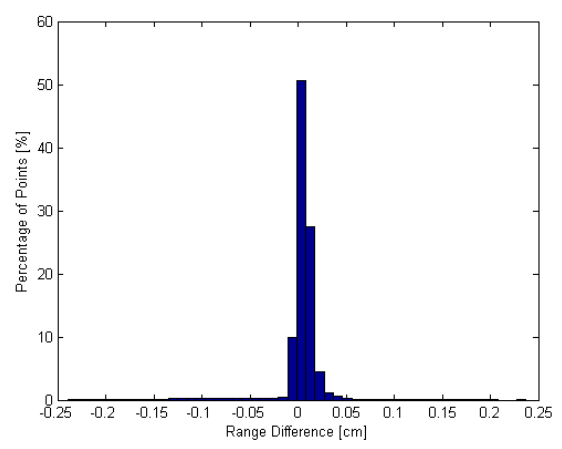

Figure 4. Histogram of range difference between the synthetic and real scan (background points in either scan are excluded)

The difference between the synthetic scan and the physical scan data is depicted in the range difference image shown in Figure 3. The blue pixels reflect background in both PCD sets. The range differences between the two datasets are represented in grey scale. Perfectly matched areas are represented in black pixels, while areas with extreme differences are shown in white pixels. The extreme difference (white pixels) in the right side of Figure 4 results from modeling inaccuracy, because in the 
'as-damaged' model, the beam was broken into only three parts, whereas in reality the right part is progressively bent and could be divided into more pieces. The more detailed the model is, the more similar the model and physical specimen are. Nevertheless, the resulting PCD are perfectly matched in the other parts, which demonstrates the validness of the computational procedure. From the histogram, shown in Figure 4, it is estimated that $95 \%$ of the points are well matched (differences are less than $0.5 \mathrm{~mm}$, and background points are not taken into account). Additional tests have been performed with models of two full-scale buildings, yielding very satisfactory results (Ma et al. 2014).

\section{VALIDATION OF SUITABILITY FOR R\&D}

The motivation for developing the computational procedure was to provide specimens for 'Scan-to-BIM' and change detection research. In order to validate the suitability of the resulting datasets to substitute for physical experiments, we compile the 'as-damaged' 3D model from both the physical and synthetic scans using a heuristic algorithm, and then compare the similarity of the two resulting models.

The algorithm starts by extracting planar segments from the PCD, then assembles the planar segments to 3D solids using information from the 'as-made' BIM model (section shape data). Segments that share edges are identified as connected segments. Then, the min-max bounding box is identified based on pairs of connected and perpendicular faces. The 'as-damaged' model (in red) compiled from the synthetic scan is compared with the 'as-built' model (in black) of the beam in Figure 5.

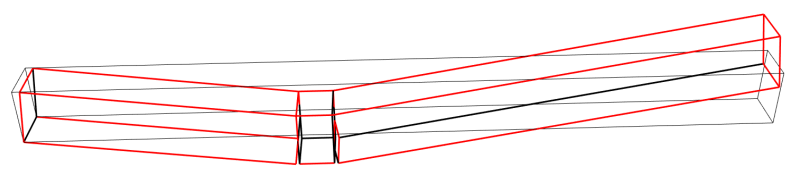

Figure 5. Comparison of 'as-damaged' and 'as-built' models.

By analyzing the model changes, the beam deflection can be derived as the projected distance from the lowest point in the 'as-damaged' model to the bottom surface of the 'as-built' model. In this case the deflection was $182 \mathrm{~mm}$. A dimensional comparison of the system generated and user prepared 'as-damaged' model is shown in Table 1, which reflects that using the heuristic algorithm for reconstruction of the 'as-damaged' beam model is very promising but still needs improved.

Table 1. Comparison of the system generated and user prepared 'as-damaged' model (all dimensions are $\mathbf{m m}$ units).

\begin{tabular}{ccccccc}
\hline Segment & \multicolumn{3}{c}{ System generated model } & \multicolumn{3}{c}{ User prepared model } \\
\cline { 2 - 7 } ID & Length & Width & Height & Length & Width & Height \\
\hline 1 & 1,378 & 234 & 292 & 1,099 & 240 & 300
\end{tabular}




\begin{tabular}{ccccccc}
2 & 280 & 234 & 292 & 163 & 240 & 300 \\
3 & 2,276 & 234 & 292 & 2,638 & 240 & 300 \\
\hline
\end{tabular}

\section{CONCLUSION}

R\&D of change detection systems for buildings is often hampered by a lack of testing specimen and data. A computational procedure using BIM and a laser scanning emulator was developed for preparing synthetic BIM and PCD specimens for the R\&D of systems for rapid change or damage detection among the changing states of a building. The procedure was validated by comparing the physical and synthetic BIM and PCD datasets. Tests for the case of earthquake damage assessment demonstrate the feasibility and the advantages of using this procedure to substitute computer modeling for costly physical experiments. An additional advantage of the computational procedure to prepare specimens for scan to BIM research is that the benchmark, i.e., the user-prepared BIM model of the digital specimen, is available for testing the system generated result, which greatly facilitates system validation.

\section{ACKNOWLEDGMENT}

This research was funded in part by the Insurance Companies Association of Israel. Dr. Ma is supported in part at the Technion by a fellowship from the Israel Council for Higher Education. The assistance of undergraduate research assistant Ashrant Aryal is greatly appreciated.

\section{References}

Akinci, B., F. Boukamp, C. Gordon, D. Huber, C. Lyons, and K. Park. 2006. "A Formalism for Utilization of Sensor Systems and Integrated Project Models for Active Construction Quality Control." Automation in Construction 15 (2): 124-38

Bosché, F., A. Guillemet, Y. Turkan, C. Haas, and R. Haas. 2014. "Tracking the Built Status of MEP Works: Assessing the Value of a Scan-vs-BIM System." Journal of Computing in Civil Engineering 28 (4): 05014004. doi:10.1061/(ASCE)CP.1943-5487.0000343.

BuildingSmart. 2013. "Industry Foundation Classes Release 4 (IFC4)." http://www.buildingsmart-tech.org/ifc/IFC4/final/html/index.htm.

Conte, Joel P, J Enrique Luco, JI Restrepo, Frieder Seible, and Lelli Van Den Einde. 2004. "UCSD-NEES Large High Performance Outdoor Shake Table." In .

Eastman, C.M., P. Teicholz, R. Sacks, and K. Liston. 2011. BIM Handbook: A Guide to Building Information Modeling for Owners, Managers, Architects, Engineers, Contractors, and Fabricators. Hoboken, NJ: John Wiley and Sons. 
German, S., J. Jeon, Z. Zhu, C. Bearman, I. Brilakis, R. DesRoches, and L. Lowes. 2013. "Machine Vision-Enhanced Postearthquake Inspection." Journal of Computing in Civil Engineering $27 \quad$ (6): 622-34. doi:10.1061/(ASCE)CP.1943-5487.0000333.

Kasai, Kazuhiko, Hiroshi Ito, Yoji Ooki, Tsuyoshi Hikino, Koichi Kajiwara, Shojiro Motoyui, Hitoshi Ozaki, and Masato Ishii. 2010. "Full-Scale Shake Table Tests of 5-Story Steel Building with Various Dampers." In .

Kashani, A., P. Crawford, S. Biswas, A. Graettinger, and D. Grau. 2014. "Automated Tornado Damage Assessment and Wind Speed Estimation Based on Terrestrial Laser Scanning.” Journal of Computing in Civil Engineering, May, 04014051. doi:10.1061/(ASCE)CP.1943-5487.0000389.

LEICA. 2014. "Leica ScanStation C10 - The All-in-One Laser Scanner for Any Application - Leica Geosystems - HDS." Accessed October 6. http://hds.leica-geosystems.com/en/Leica-ScanStation-C10_79411.htm.

Ma, L, R Sacks, and R Zeibak-Shini. 2014. "Information Modeling of Earthquake-Damaged Reinforced Concrete Structures." Advanced Engineering Informatics.

Ma, L, R Sacks, R Zeibak-Shini, A Aryal, and S Filin. 2014. "Preparation of Synthetic 'As-Damaged' Models for Post-Earthquake BIM Reconstruction Research." Journal of Computing in Civil Engineering.

Panagiotou, Marios, José I Restrepo, and Joel P Conte. 2010. "Shake-Table Test of a Full-Scale 7-Story Building Slice. Phase I: Rectangular Wall." Journal of Structural Engineering 137 (6): 691-704.

Schwarz, S., A. Hanaor, and D. Yankelevsky. 2008. Horizontal Load Resistance RC Frames with Masonary Infill Panels. National Building Research Institute, Technion.

Tagawa, Y, and K Kajiwara. 2007. "Controller Development for the E-Defense Shaking Table." Proceedings of the Institution of Mechanical Engineers, Part I: Journal of Systems and Control Engineering 221 (2): 171-81.

Torok, M., M. Golparvar-Fard, and K. Kochersberger. 2014. "Image-Based Automated 3D Crack Detection for Post-Disaster Building Assessment." Journal of Computing in Civil Engineering 28 (5): A4014004. doi:10.1061/(ASCE)CP.1943-5487.0000334.

Van de Lindt, John W, Shiling Pei, Steven E Pryor, H Shimizu, and H Isoda. 2010. "Experimental Seismic Response of a Full-Scale Six-Story Light-Frame Wood Building.” Journal of Structural Engineering 136 (10): 1262-72.

Zeibak, R., and S. Filin. 2007. "Change Detection via Terrestrial Laser Scanning." International Archives of Photogrammetry and Remote Sensing 36 (3/W52): $430-35$. 